\title{
MACROMIXING EFFECTS ON THE GRAY-SCOTT MODEL IN A STIRRED REACTOR
}

\author{
T. J. HSU, ${ }^{B}$ C. Y. MOU ${ }^{\mathbb{a}}$ and D. J. LEE ${ }^{\mathrm{b} *}$ \\ Department of Chemistry, \\ ${ }^{b}$ Department of Chemical Engineering, National Taiwan University, Taipei, Taiwan, 106, ROC
}

\begin{abstract}
A numerical investigation of the macromixing effects on the Gray-Scott model in a stirred tank was undertaken based on a global fluid flow field model. With a high mixing intensity, all zones in the tank would resemble that of a perfect CSTR. When mixing intensity is decreased, both the stationary and oscillatory states are affected. The major role of the macromixing is found to alter the relative stability between the stationary and the oscillatory states.
\end{abstract}

\section{INTRODUCTION}

Mixing may alter the complex system dynamics both quantitatively and qualitatively (Villermaux, 1991, Dutt and Menzinger, 1992). The importance of mixing is usually discussed in terms of macromixing and micromixing. Macromixing effects on complex chemical dynamic systems has been investigated in several studies (Kumpinsky and Epstein, 1985, Eli-Bar and Noyes, 1986, Gyorgyi and Field, 1989a, 1989b, Ganapathisubramanian, 1991, Hauser et al., 1992).

Various geometrical factors including reactor shape, stirrer type or the existence of baffles all affect the macromixing significantly (Ganapathisubramanian, 1991). Some works discussed the macromixing effects based on the global fluid flow field in the stirred tank (Hsu et al., 1994, Hsu and Lee, 1995). They showed that the incomplete mixing might generate apparent complex oscillations from an oscillator exhibiting only simple limit cycle behaviour (the Oregonator model for $\mathrm{BZ}$ reaction), or change the attraction basin for the bistable chemical system (the Kumpinsky-Esptein model for the chlorite-iodide reaction). No comprehensive analysis on the possible role of macromixing on a chemical system exhibiting both bistable and oscillatory characters had been reported based on the global fluid flow field.

The Gray-Scott (G-S) model is one of the simplest nonlinear kinetic models exhibiting both the bistable and oscillatory dynamic behaviour (Gray and Scott, 1983, 1984, 1985). It's an abstract scheme but reveals key aspects of many real systems (Middya and Luss, 1994). The purpose of the present work is to investigate the effects of macromixing on the relative stability of the bistable and the oscillatory attractors for the G-S model. The so-called "well-mixing limit " is addressed as well.

\section{THE MODELS}

The G-S model corresponds to the chemical reactions as follows:

$$
\begin{aligned}
& u+2 v \stackrel{k_{1}}{\rightarrow} 3 v, \\
& v \stackrel{k_{2}}{\rightarrow} p .
\end{aligned}
$$

In a perfect CSTR, with feed of pure reactant $u\left(u_{0}\right)$, the dynamic mass balance equations can be stated as follow:

$$
\begin{aligned}
& \frac{d u}{d t}=-k_{1} u v^{2}+\frac{q}{V_{m}}\left(u_{0}-u\right), \\
& \frac{d v}{d t}=k_{1} u v^{2}-k_{2} v+\frac{q}{V_{m}}(0-v) .
\end{aligned}
$$

With the dimensionless groups defined as follows:

$$
U=u / u_{0} ; V=v / u_{0} ; F=q / V_{m} k_{1} u_{0}^{2} ; k=k_{2} / k_{1} u_{0}^{2} ; \tau=t k_{1} u_{0}^{2},
$$

eqs. (3) and (4) can be rearranged into the following dimensionless equations (Pearson, 1993):

$$
\frac{d U}{d \tau}=-U V^{2}+F(I-U) \text {, }
$$




$$
\frac{d V}{d \tau}=U V^{2}-(F+k) V
$$

where $U$ and $V$ are the reactants, $F$ the reduced flow rate, $k$ the reduced rate constant, and $\tau$ the dimensionless time. The steady states for $U$ are 1.0 (washout steady state), $\left(1+\sqrt{1-4(F+k)^{2} / F}\right) / 2$ (middle steady state) and $\left(1-\sqrt{1-4(F+k)^{2} / F}\right) / 2$ ) (thermodynamic steady state). Clearly the last two steady states could exist only if the sign of the group $4(F+k)^{2}-F$ is negative. The set with this group as zero forms the saddle-node (SN) bifurcation curve.

Figure 1 shows the bifurcation diagram for the G-S model under perfect CSTR limit (Pearson, 1993). The solid curve and the dashed curve represent the lower SN and the Hopf-bifurcation curves, respectively. Within the solid (SN) curve enveloped region, all three steady states described above coexist with the middle one unstable. Outside the enveloped region, only the washout steady state can exist. Between the lower SN and the Hopf-bifurcation curves, stable limit cycle can occur when $k<0.035$. If $k$ is larger than 0.035 , an unstable limit cycle will occur.

The macromixing model employed in the present study was the networks-of-zones model, which was originally developed by Mann and co-workers (for a brief literature review, see Nienow et al. (1992)). The details of the parameters of the flow model can be found elsewhere (Hsu, et al., 1994). Basically the stirred tank is assumed to comprise $2 \times \mathrm{NxN}$ zones whose volume is set as unity. Each zone has a through-flow (flow rate of $Q_{1}$ ) and two side-flow streams (each with a flow rate of $0.2 \cdot Q_{i}$ ) between its nearest adjacent zones. A total of $2 \times 2 \times N \times N$ ODEs are thereby resulted and required to solve numerically (in most cases $N$ was set as 10 ). Gear method was employed as the integration tool with a relative error less than $10^{-7}$. The total number of job execution was beyond 1450 .

\section{RESULTS AND DISCUSSION}

\section{High internal flow rate}

When the internal flow rate is high $\left(Q_{i}>500\right.$, with an exchange flow rate of $\left.0.2 \times 500=100\right)$, the concentrations of all zones would evolve simultaneously as those in a perfect CSTR. No global heterogeneity is observed over the whole tank. Outside the SN curve enveloped region in Fig. 1, all zones would go to the washout steady state (flow branch). Within the enveloped region by the upper $\mathrm{SN}$ and the Hopf-bifurcation curves, bistability occurs. Figure 2 gives a calculation example with $k=0.05$ and $Q_{i}=1000$ (bold curve). Clearly the basin of attraction for the thermodynamic steady state (T-branch) is strongly affected by both the parameter $F$ and the initial condition $V_{\text {intir }}$. The final steady states for all zones are either 1.0 (flow branch) or the F-branch predicted at the perfect CSTR limit. Notably the margins for the bistable region would depend on the initial conditions chosen.

In the vicinity of Hopf-bifurcation curve shown in Fig. 1, stable limit cycle behaviour is also found for the networks-of-zones model with $Q_{i}=1000$. The oscillatory amplitude and the corresponding oscillatory period are all the same as those in a perfect CSTR.

Both the stationary and oscillatory characteristics for the macromixing model with a $Q_{i}$ larger than 300 are thereby consistent with those for a perfect CSTR. As a result, the stirred reactor can be taken as a perfect CSTR Interestingly, this threshold $Q_{j}$ value is different from that for the Oregonator model (50, Hsu et al., 1994) or for the Kumpinsky-Epstein model (20000, Hsu and Lee, 1995). That is, the so-called "well-mixing limit" for a reactor is actually dependent on the chemical reactions involved. This puts a precaution on the application of the widely employed tracer test for the diagnosis of the degree of mixing in a reactor (Levenspiel, 1972).

\section{Intermediate internal flow rate}

Global heterogeneity occurs over the whole tank as the internal flow rate decreases. In the bistable region both the locations and range for the attraction basin of $\mathrm{T}$-branch change. The marginal stability curves for $Q_{i}=200$ and $k=0.05$ is shown as a dot-dashed curve in Fig. 2 . Within the attraction basin for T-branch, the phase point will be attracted spirally towards the thermodynamic steady state. Outside the basin the final state is the washout steady state. Mapping the margins for the bistable region onto the bifurcation diagram as that in Fig. 3 in Hsu and Lee (1995), the effect of incomplete macromixing is to stabilize rather than destabilize the T-branch. This is in contrast to that found for the Kumpinsky-Epstein bistable model (Hsu and Lee, 1995). Incomplete macromixing thereby tends to destabilize the T-branch if the steady state is a stable node, or stabilize it if the steady state is an unstable focus. The net effect is to shift the attraction basin for T-branch to the region of lower parameter $F$ and higher $V_{\text {trir }}$

The SN-bifurcation curves for the present networks-of-zones model is hard to determined analytically or numerically (the Hopf-bifurcation curve would be first touched when the operating point is moving vertically downward in Fig. 1). For a given parameter $F$, the critical $k$ value below which the stable focus has changed into 


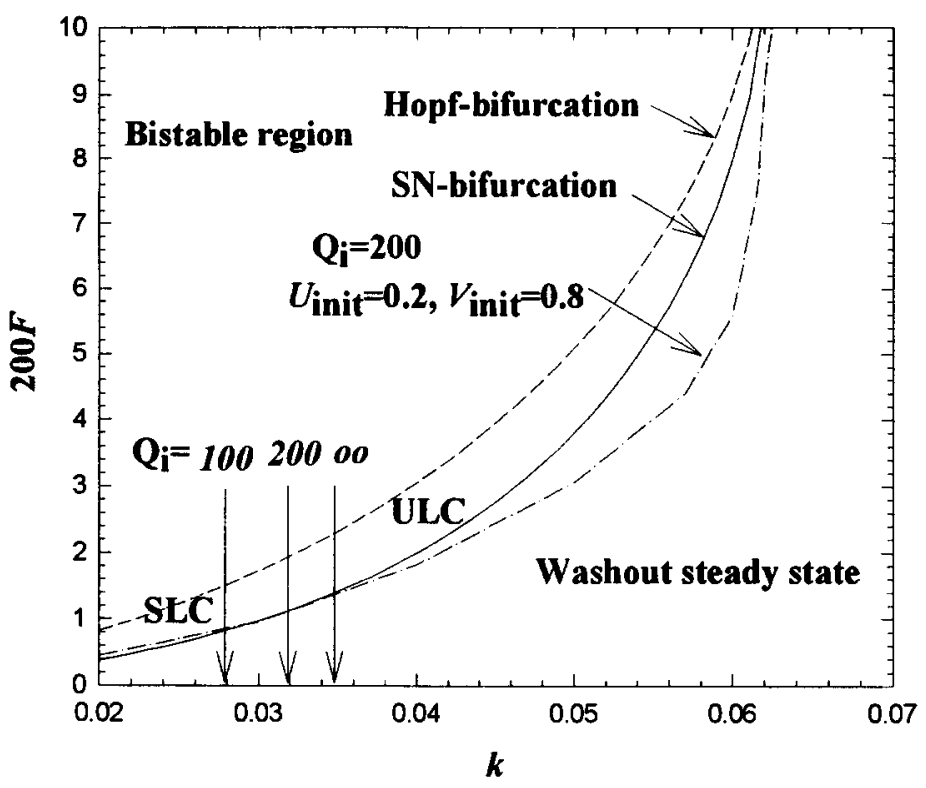

Fig. 1 Phase diagram of the Gray-Scott model.

SLC:stable limit cycle; ULC:unstable limit cycle.

Vertical arrows: critical $k$ value divided SLC and ULC.

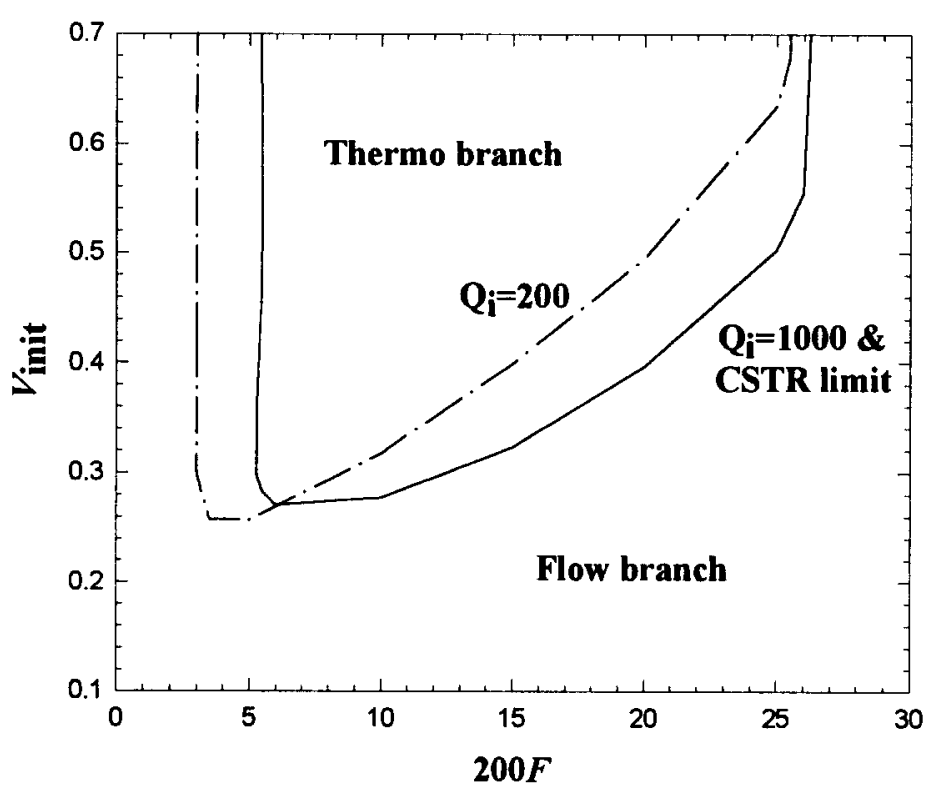

Fig. 2 Attraction basin for $\mathrm{T}$ and $\mathrm{F}$ branches. $U_{\text {init }}=0.2, k=0.05$. 


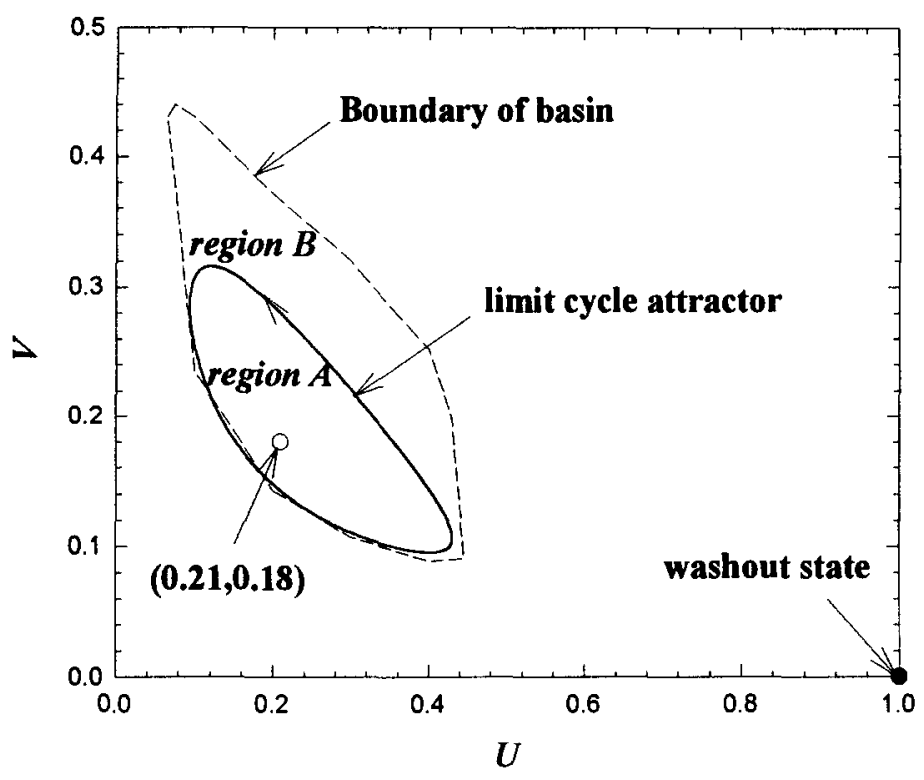

Fig. 3 Limit cycle attractor for Gray-Scott model under incomplete macromixing. $U_{\text {init }}=0.2, k=0.03,200 F=0.993$.

unstable focus is determined via comprehensive numerical works. The marginal stability curve thus determined can be taken as the Hopf-bifurcation for the system. The results are also demonstrated as the dot curve in Fig. 1. Notably, the unstable region between the lower SN and Hopf-bifurcation curve under perfect mixing limit is stabilized as the internal flow rate decreases. Or equivalently, the Hopf-bifurcation curve has been pushed downwards and rightwards in Fig. 1.

Stable limit cycle behaviour can be detected for $Q_{i}=200$ when $k$ is less than 0.032 . Since the concentrations for all zones are different, the mean value is considered. One example is given in Fig. 3. The unstable focus and the boundary of the attraction basin (dashed curve) are located via numerical results. The threshold $k$ value less than which the stable limit cycle can be detected for $Q_{i}=200$ or 100 is 0.032 or 0.028 respectively (compared with the value of 0.035 for perfect CSTR condition). Incomplete macromixing would therefore tend to destabilize the stable limit cycle behaviour for the G-S model.

Interestingly, the increasing of the oscillation period is also found for the G-S model, which is similar to that for the Oregonator model (Hsu et al., 1994).

\section{Low internal flow rate}

Decreasing the internal flow rate still further $\left(Q_{i}<35\right)$ would cause the bistable region to become even larger on the stability diagram in Fig. 1. The attraction basin for the oscillatory attractor shrinks also to make the oscillatory region more difficult to be located. Complex oscillations occur in the vicinity of the Hopf-bifurcation curve. The pattern is similar to that for the Oregonator model (Hsu et al., 1994).

\section{Relative stability between stationary and oscillatory states}

Simulation results demonstrate that, for the stationary states, a reduction in internal flow rate would stabilize the thermo steady state from becoming an unstable focus, but would have almost no effects on the flow branch. The net effect is to extend the bistable region on the bifurcation diagram. This is not consistent with the conclusions drawn from the bistable K-E model (Hsu and Lee, 1995). On the oscillatory states, the macromixing tends to destabilize the limit cycle behaviour or to shrink the attraction basin of the oscillatory attractor. The oscillation period is also found to reduce when the mixing intensity is reduced. This is consistent with the results for Oregonator model (Hsu et al., 1994). These results suggest that the major effects of macromixing is to alter the relative stability between the thermodynamic steady state and the oscillatory states.

Hauser et al. (1992) demonstrated that the early or late mixing of reactants (by changing the direction of the stirrer) can alter the relative stability between the stationary and the oscillatory states for the minimal Bromate system under non-premixed feed condition. The Hopf-bifurcation curves are shifted accordingly. Foerster et al. (1993) first measured the relative stability between two stationary states. In the present G-S model, the location 
of the upper SN-bifurcation and the intersection point between the Hopf-bifurcation curve is little affected by the macromixing. Nevertheless, the location of the Hopf-bifurcation is strongly influenced.

From the data in Fig. 3 and other numerical results, although the location of the attraction basin is shifted, the relative stability between the thermodynamic and the washout steady state is basically unchanged by the internal flow rate. On the relative stability between the oscillatory attractor and the washout steady state, a series numerical calculations have been conducted as follows. The initial condition for zones on the upper three rows in networks-of-zones model were set as the washout steady state $(1,0)$, while those in the lower part were somewhere in region A or B in Fig. 3. In most cases, the final state for all zones would be $(1,0)$ when the lower zones were in region $B$ initially. Only when the initial conditions were in region $A$, the final state could have chance to be attracted onto the limit cycle. Clearly the stationary state is relatively more stable under incomplete macromixing conditions.

\section{CONCLUSIONS}

When the internal flow rate is high $\left(Q_{i}>500\right)$, all zones evolve simultaneously as in a perfect CSTR. No global heterogeneity is observed while the stationary and the oscillatory characteristics are the same as those under well the mixing limit. As the internal flow rate is decreased, the thermodynamic steady state is stabilized while the limit cycle attractor is destabilized. The major effect of macromixing is to alter the relative stability between the stationary and the oscillatory states.

ACKNOWLEDGEMENTS- The authors want to thank the financial support from National Science Council, R.O.C. The numerical works were performed on the IBM SP2 cluster in the National Center for High-Performance Computing, NSC, Taiwan.

\section{NOTATIONS}

$F \quad=$ dimensionless feed flow rate defined in eq. (5), -

$k \quad=$ reduced rate constant defined in eq. (5), -

$k_{1} \quad=$ rate constant, $\mathrm{M}^{-2} \mathrm{~s}^{-1}$

$k_{2} \quad=$ rate constant, $\mathrm{s}^{-1}$

$N \quad=$ parameter in networks-of-zones model

$Q_{i} \quad=$ dimensionless internal through flow rate, -

$q \quad=$ reactant flow rate, $\mathrm{m}^{3} / \mathrm{s}$

$t \quad=$ time, $\mathrm{s}$

$U \quad=$ dimensionless concentration of reactant $u$, -

$U_{\text {init }} \quad=$ initial concentration for reactant $U$, -

$u \quad=$ reactant, $\mathbf{M}$

$V \quad=$ dimensionless concentration of reactant $v$,

$V_{\text {init }} \quad=$ initial concentration for reactant $V$, -

$V_{m} \quad=$ reactor volume, $\mathrm{m}^{3}$

$v \quad=$ reactant, $\mathrm{M}$

$\tau=$ dimensionless time defined in eq. (5), -

\section{REFERENCES}

Dutt, A. K. and Menzinger, M., 1992, Effect of stirring and temperature on the Belousov-Zhabotinskii reaction in a CSTR. J. Phys. Chem. 96, 8447-8449.

Eli-Bar, K. and Noyes, R. M., 1986, A model for imperfect mixing in a CSTR. J. Chem. Phys. 85, 3251-3257.

Foerster, P., Zhang, Y. and Ross, J., 1993, Experiments on relative stability in the bistable multivariable bromate-ferroin reaction. J. Phys. Chem. 97, 4708-4713.

Ganapathisubramanian, N., 1991, Tristability in the iodate/As(III) chemical system arising from a model of stirring and mixing effects. J. Chem. Phys. 95, 3005-3008.

Gray, P. and Scott, S. K., 1983, Autocatalytic reactions in the isothermal, continuous stirred tank reactor. Isolas and other forms of multistability. Chem. Engng. Sci., 38, 29-43.

Gray, P. and Scott, S. K., 1984, Autocatalytic reactions in the isothermal, continuous stirred tank reactor. Oscillations and instabilities in the system A+2B $\rightarrow 3 B$; B $\rightarrow$ C. Chem. Engng. Sci., 39, 1087-1097.

Gray, P. and Scott, S. K., 1985, Sustained oscillations and other exotic patterns of behavior in isothermal reactions. J. Phys. Chem., 89, 22-32.

Gyorgyi, L. and Field, R. J., 1989a, Aperiodicity resulting from two-cycle coupling in the Belousov-Zhabotinskii reaction 2. Modeling of the effect of dead spaces at the input ports of a continuous-flow stirred tank reactor. J. Phys. Chem. 93, 2865-2869. 
Gyorgyi, L. and Field, R. J., 1989b, Aperiodicity resulting from two-cycle coupling in the Belousov-Zhabotinskii reaction. III. Analysis of a model of the effect of spatial inhomogeneities at the input ports of a continuous-flow, stirred tank reactor, J. Chem. Phys. 91, 6131-6141.

Hauser, M. J. B., Lebender, D. and Schneider, F. W., 1992, Stirring sense discriminates between stationary and oscillatory states. J. Phys. Chem. 96, 9332-9338.

Hsu, T. J. and Lee, D. J., 1995, Macromixing effects on the Kumpinsky-Epstein model of a chlorite-iodide reaction in a stirred tank. J. Chem. Phys. 102, 8274-8276.

Hsu, T. J., Mou, C. Y. and Lee, D. J., 1994, Effects of macromixing on the Oregonator model of the Belousov-Zhabotinsky reaction in a stirred tank. Chem. Engng. Sci. 24B, 5291-5305.

Kumpinsky E; Epstein I. R., 1985, A model for stirring effects on transitions in bistable chemical systems. $J$. Chem. Phys. 82, 53-57.

Levenspiel, O., 1972, Chemical Reaction Engineering. John Wiley \& Sons, New York.

Middya, U. and Luss, D. 1994, Impact of global interactions on patterns in a simple system. J. Chem. Phys. 100, 6386-6394.

Nienow, A. W., Drain, S. M., Boyen, A. P., Mann, R. and El-Hampoz, A. M., 1992, A new pair of reactions to characterize imperfect macromixing and partial segregation in a stirred semi-batch reactor. Chem. Engng. Sci. 47, 2825-2830.

Pearson, J. E., 1993, Complex patterns in a simple system. Science. 261, 189-192.

Villermaux, J., 1991, Mixing effects on complex chemical reactions in a stirred reactor. Rev. Chem. Engng., 7 , 51-108. 